\title{
Uso de sistemas de información geográfica (SIG) en la valoración del potencial piscícola a nivel municipal
}

\author{
Use of geographic information systems (GIS) for the assessment of \\ fish farming potential at the municipal level
}

\section{Uso dos sistemas de informação geográfica (SIG) na valoração do potencial para piscicultura ao nível municipal}

\author{
Sandra C Pardo-Carrasco ${ }^{1}$ \\ 1 Universidad Nacional de Colombia Sede Medellín, FCA/DPA/BIOGEM. \\ Email:scpardoc@unal.edu.co
}

Recibido: 25 de julio de 2015

Aceptado: 18 de agosto de 2017

\begin{abstract}
Resumen
La aplicación de los Sistemas de Información Geográfica (SIG) en diversas áreas del conocimiento ha experimentado un gran desarrollo en las últimas dos décadas, especialmente para resolver problemas en la toma de decisiones y la organización de un territorio determinado. Es así como los SIG en piscicultura, han sido especialmente utilizados en la determinación del potencial de cualquier área para el desarrollo de sistemas de cultivo. Además, sirven como una herramienta para dar recomendaciones a los procesos de ordenación y planificación de la actividad. El municipio Castilla La Nueva, ubicado en el departamento del Meta, se seleccionó como área de estudio porque allí la piscicultura constituye un renglón económico importante dentro del sector agrícola. Debido a los efectos ambientales adversos que la piscicultura puede producir (contaminación de agua, cambios de usos del suelo, conflictos de usos, etc.) cuando se realiza sin planificación y orden, se hace necesario introducir técnicas modernas en el análisis de información, para contribuir con la planificación municipal e integrar de forma coherente la piscicultura con el entorno y otras actividades presentes. Los resultados obtenidos con la aplicación del SIG basado en el modelamiento matemático-estadístico están representados por cartografía temática y un mapa de aptitud piscícola de la zona de estudio. Se espera que dichos resultados contribuyan a la identificación de los lugares más aptos para el desarrollo de la piscicultura en Castilla La Nueva, para que así los planificadores del municipio tengan bases adecuadas para organizar el sector rural con mayor conocimiento y compromiso ambiental. También que este sea un ejemplo a seguir para el ordenamiento territorial a nivel de municipios en Colombia.
\end{abstract}

Palabras clave: SIG, piscicultura, potencialidad, ordenamiento territorial, planificación

\begin{abstract}
There has been a great deal of development in the use of geographic information systems (GIS) in a variety of areas during the past two decades, particularly for decision-making related to regional planning. In fish farming, GIS have been used particularly to determine site potential. Furthermore, they can be used as a tool to provide recommendations for the planning and implementation stages of fish farming projects. The Castilla La Nueva municipality, located in the Meta department in
\end{abstract}


Colombia, was chosen for this study because fish farming activities are an important economic portion of the agriculture sector. Due to the adverse environmental effects, that fish farming may produce when not properly implemented (water pollution, land-use change, land-use conflicts, etc.), the modern information analysis techniques becomes critical for municipal planning tasks such as the integration of fish farming and environmental conservation. The results obtained after implementing the GIS, which was based on mathematical and statistical modeling, are represented by thematic mapping and a map of the studied zone displaying the area's suitability for fish farming. We expect that these results will contribute to the identification of the most suitable places for fish farming in Castilla La Nueva. This will provide the municipality's town planners with the elements required to organize the rural sector with more knowledge and environmental commitment. Likewise, we also hope that this will set an example to be followed by other Colombian municipalities in terms of zoning.

Key words: GIS, fish farming, potential, land, planning

\begin{abstract}
Resumo
O uso dos Sistemas de Informação Geográfica (SIG) a diversas áreas do conhecimento tem experimentado um grande desenvolvimento nas últimas dois décadas, especialmente para resolver problemas na toma de decisões e a organização de um determinado território. É assim como os SIG em piscicultura, tem sido especialmente utilizados na determinação do potencial de qualquer área para o desenvolvimento de sistemas de cultivo. Além disso, servem como uma ferramenta para dar recomendações aos processos de ordenamento e planejamento da atividade. O município Castilla La Nueva, localizado no departamento do Meta, foi selecionado como área de estudo porque ali a piscicultura se constitui numa parte económica importante dentro do setor agrícola. Devido aos efeitos ambientais adversos que a piscicultura pode produzir (poluição da água, câmbios de usos do solo, conflitos de usos, etc.) quando se realiza sem planejamento nem ordem, é necessário introduzir técnicas modernas na análise de informação, para contribuir com a planificação do município e integrar de forma coerente a piscicultura com o entorno e outras atividades presentes. Os resultados obtidos com a aplicação do SIG baseado na modelagem matemático-estatístico estão representados pela cartografia temática e um mapa de atitude piscícola da zona de estúdio. Espera-se que ditos resultados contribuam à identificação dos locais mais aptos para o desenvolvimento da piscicultura em Castilla La Nueva, para que assim os planejadores do município tenham suporte adequado para organizar o setor rural com maior conhecimento e compromisso ambiental. Também que este seja um exemplo para ser seguido no ordenamento territorial ao nível de municípios na Colômbia.
\end{abstract}

Palavras chave: SIG, piscicultura, potencialidade, ordenamento territorial, planejamento

\section{Introducción}

Uno de los sistemas de producción animal con mayor tasa de crecimiento anual en las últimas décadas es la acuicultura, actividad a la cual se le reconocen ventajas para aliviar la pobreza, mejorar la seguridad alimentaria y traer desarrollo rural (FAO, 2009). Por su importancia, y porque como cualquier otra actividad tiene efectos negativos sobre el ambiente, la FAO desarrolló el Código de Conducta para la Pesca Responsable (FAO, 1995) dedicando el artículo noveno a las recomendaciones para realizar la acuicultura de forma responsable. El código resalta que la práctica de la acuicultura debe hacerse de forma sustentable para que puedan obtenerse los beneficios, y dice que la evaluación de la aptitud de los espacios en donde se implantarán los cultivos es una de las primeras tareas que debe ejecutarse. En este sentido, el Código promueve la aplicación de Sistemas de Información Geográfica (SIG) para estas evaluaciones, siendo una herramienta muy importante al momento de resolver problemas de carácter espacial, ya que por medio de la recolección, proceso y análisis de una serie de datos biofísicos y socioeconómicos relacionados directamente con las necesidades de la actividad piscícola, puede producir mapas con las áreas de mayor voca- ción (Kapetsky y Nath, 1997; Nath et al., 2000; Seiffert, 2004). De esta forma, pueden darse recomendaciones para la ordenación y planificación de cualquier actividad que pueda entrar en conflicto con el medio ambiente; tanto así que con el paso del tiempo se han venido utilizando interdisciplinariamente en diferentes áreas de la investigación.

Los SIG, además de una correcta planificación, permiten hacer monitoreo de la acuicultura, así como evaluar sus posibles impactos ambientales (AguilarManjarrez et al., 2006). Adicionalmente ayudan en la toma de decisiones respecto al uso que debe ser dado a la tierra (Nath et al., 2000) y posibilitan evaluar la vocación de varias áreas en forma rápida y sistemática (Giap et al., 2005). De acuerdo con Kapetsky y Travaglia (1995), los SIG permiten que el inversionista interesado en acuicultura tenga información espacial para la selección del área y del sistema de producción que es más favorable implementar.

Existen varios ejemplos de uso de SIG en acuicultura, desde estudios en grandes áreas y de baja resolución como en Latinoamérica (Kapetsky y Nath, 1997) y África (Aguilar-Manjarrez y Nath, 1998), áreas medianas y 
resolución media en países como Zimbawe (Chimowa y Nugent, 1993) y Ghana (Kapetsky et al., 1990), departamentos o estados como Tabasco y Sinaloa en México (Aguilar-Manjarrez y Ross, 1992; AguilarManjarrez y Ross, 1995) y pequeñas áreas y con alta resolución como la bahía de Sepetiba en Brasil (Scout et al., 1998) y la bahía de Ruiadhe en Escocia (Ross et al., 1993). Su uso se ha enfocado principalmente a tres aplicaciones, selección de zonas óptimas para el desarrollo de esta actividad, modelos dinámicos y estudios de series temporales. En este caso, se usó el SIG para evaluar la aptitud piscícola del municipio de Castilla La Nueva, Meta (Colombia).

La actividad piscícola ocupa un lugar importante dentro de la economía del municipio de Castilla La Nueva, así como la práctica de otras actividades como el cultivo de palma y arroz, ganadería y extracción petrolera. Por esto es necesario identificar las zonas más aptas para la piscicultura y poderla integrar de forma coherente con su entorno y demás actividades económicas; además para que los planificadores del municipio cuenten con una base sólida para organizar el sector rural con mayor conocimiento.

Es importante resaltar que la localización de zonas óptimas para el desarrollo de la piscicultura es una prioridad para las administraciones a escala municipal, departamental y nacional; como medida de ordenación y de planificación para el futuro desarrollo de esta actividad. Por las razones anteriores este trabajo tuvo por objetivos estructurar un banco de datos a través del análisis de la información del municipio Castilla la Nueva - Meta y de sus bases cartográficas, para orientar el desarrollo de la piscicultura de manera sostenible, e identificar las áreas con aptitud piscícola utilizando funciones de análisis espacial y de este manera contribuir con la planificación territorial del municipio.

\section{Materiales y métodos}

\section{Área de estudio}

El área de estudio lo comprende el municipio de Castilla La Nueva, el cual se encuentra ubicado al noroccidente del departamento del Meta. Geográficamente el municipio está situado en la llanura oriental, zona adyacente al piedemonte de la Cordillera Oriental; $03^{\circ}$ $49^{\prime} 49^{\prime \prime} \mathrm{N}$ y $73^{\circ} 40^{\prime} 57^{\prime \prime} \mathrm{W}$. Cuenta con un área total de 50.728 ha, limitando al Norte con el municipio de Acacias y San Carlos de Guaroa, al Sur con San Martín, al Oriente con San Carlos de Guaroa y al Oeste con Guamal.

\section{Herramientas tecnológicas}

Este estudio fue desarrollado utilizando el software SIG ArcView 3.3 y ArcGis 9.2 con los cuales se generó toda la base cartográfica, que recoge la información temática utilizada para localizar las zonas con aptitud piscícola en el municipio. La información fue relacionada por medio de un modelamiento matemáticoestadístico y representada por cartografía temática en formato shp (shapefile), con proyección local de Colombia (sistema transversla de Mercator, Datum Observatorio de Bogotá.

La figura 1 muestra los pasos que se llevaron a cabo en el desarrollo del proyecto.

Se realizó una revisión de toda la información con la que se contó, incluyendo los datos disponibles en el Esquema de Ordenamiento Territorial de Castilla La Nueva, datos de calidad de agua para los principales drenajes del municipio, encuestas aplicadas a los productores piscícolas (Pardo-Carrasco, 2006) y por otra parte la cartografía básica y temática; dicha cartografía se obtuvo del Estudio General de Suelos del Departamento de Meta realizado por el Instituto Geográfico Agustín Codazzi, de este se utilizaron los siguientes mapas, Unidades de Paisaje que fue la unidad espacial utilizada para realizar los procesos y análisis cartográficos, Red Hidrográfica, Red Vial, Cobertura Vegetal y Usos del Suelo, Relieve, Amenaza por Inundación, Materiales del Suelo, Textura del Suelo, Fertilidad y Acidez del Suelo y Conflictos de Usos del Suelo, todos estos temas fueron indispensables para llevar a cabo el

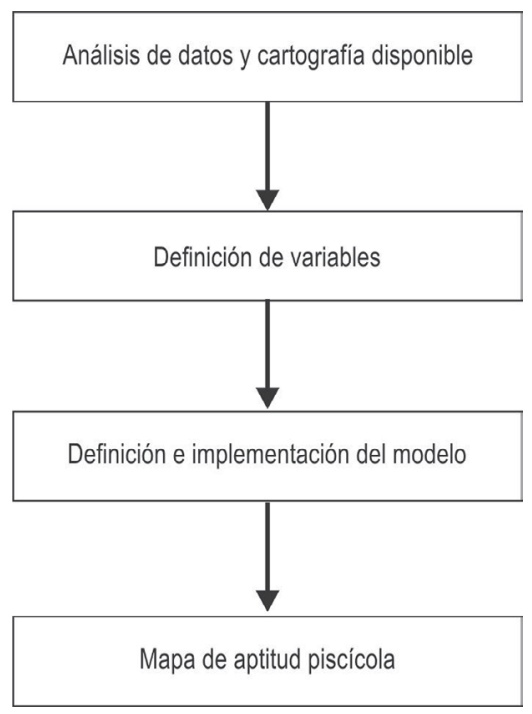

Figura 1. Pasos desarrollados en el proyecto 
objetivo del estudio; a partir de estos se pudo obtener el resto de variables presentadas en la figura 2 .

Además de utilizar ArcView y ArcGis para generar la cartografía en formato shp, se hizo necesario el uso del software para procesamiento de imágenes de satélite ERDAS 8.4 para la aplicación del modelo matemático que arrojaría los valores de aptitud. La escala de trabajo de la cartografía corresponde a 1:100000 para la escala de análisis y 1:200000 para la escala de presentación final del mapa.

\section{Identificación de variables}

Se definieron las variables importantes para el análisis del potencial piscícola de la zona de estudio (Figura 2). Para precisar las variables, primero se establecieron cuatro grandes temas (Ubicación potencial de estanques, Calidad del suelo, Calidad del agua y Socioeco- nomía e infraestructura) que pudieran recoger todos los componentes a tener en cuenta para la aptitud piscícola; tomando como base para el análisis espacial las unidades de paisaje que conforman el municipio de Castilla La Nueva y de este modo cada uno de estos grandes temas están compuestos por cada una de las variables evaluadas.

Para obtener los valores de aptitud piscícola se implementó un análisis multicriterio a través de la siguiente ecuación matemática:

$$
A P=C \times P
$$

Dónde:

$\boldsymbol{A P}=$ Aptitud piscícola

$\boldsymbol{C}=$ Calificación de la aptitud según cada variable

$\boldsymbol{P}=$ Peso, definido según la importancia de cada variable

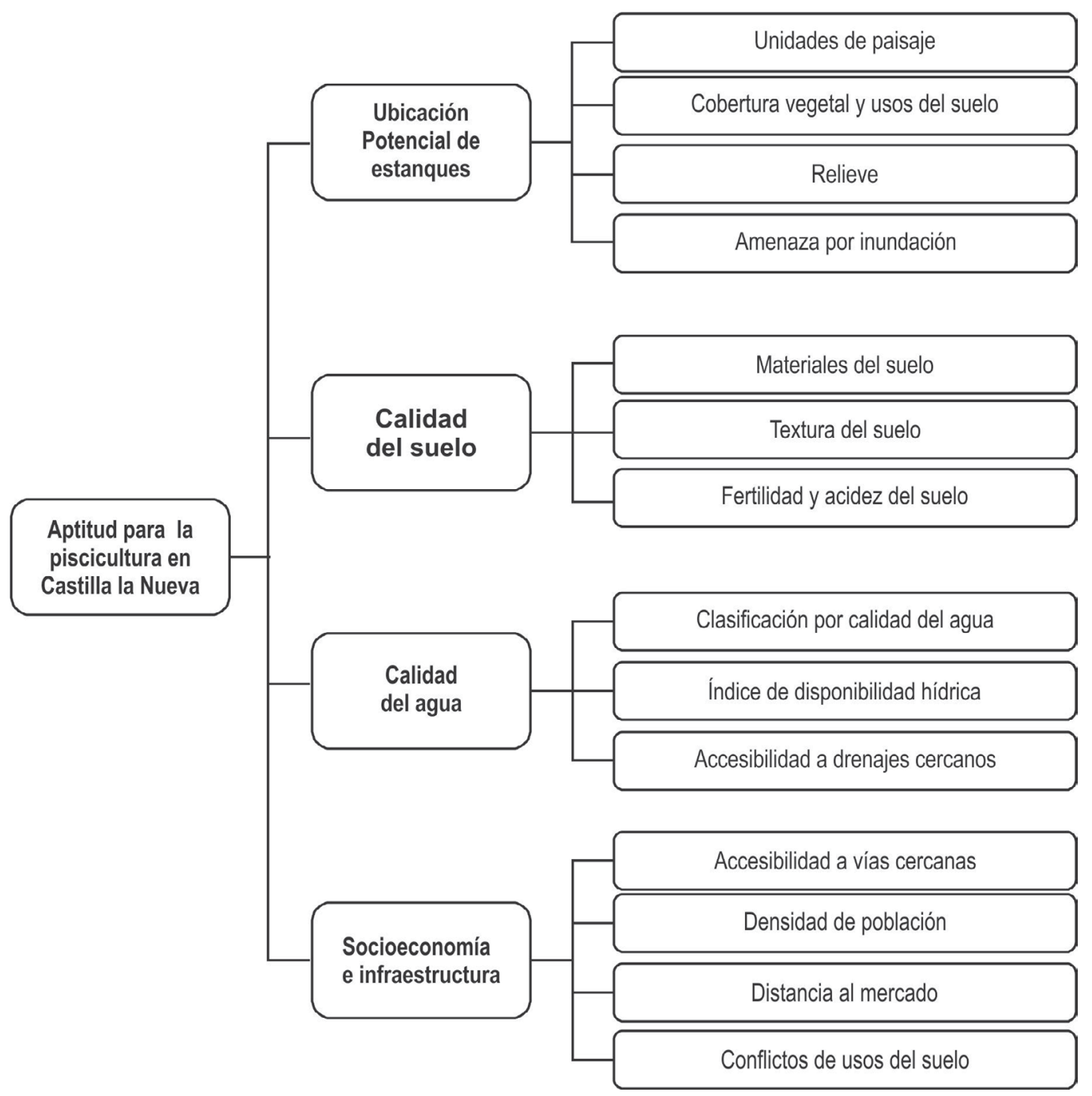

Figura 2. Agrupación de variables (Modelo simplificado del SIG) 
De acuerdo con esta ecuación, cada una de las variables inicialmente fue evaluada de forma independiente para evaluar la aptitud para cada variable, para luego relacionar los resultados de tal manera que se obtuvieron valores para los cuatro grandes grupos y por último los valores finales de la aptitud piscícola del municipio.

\section{Clasificación y evaluación}

Para llegar al resultado final primero se clasificó la aptitud de cada variable en cuatro categorías de análisis desde Apta hasta No apta (Tabla 1). Se clasificaron las variables según sus características, en esta se especificaron los elementos o condiciones que deben presentar cada una de las variables para cumplir con las cuatro categorías de aptitud.

El paso siguiente fue incorporar el peso a cada variable, expresada en forma de ponderación; esto con el propósito de cuantificar el grado de importancia de las variables con respecto a las demás que componen el modelo.

Tabla 1. Criterios para asignar la clasificación de la aptitud

\begin{tabular}{|c|c|c|c|c|}
\hline Variables & $\begin{array}{c}\text { Alta } \\
100-80\end{array}$ & $\begin{array}{c}\text { Apta } \\
<80-60\end{array}$ & $\begin{array}{l}\text { Apta baja } \\
<60-40\end{array}$ & $\begin{array}{c}\text { No apta } \\
<40\end{array}$ \\
\hline Calificación & 3 & 2 & 1 & $\mathbf{0}$ \\
\hline $\begin{array}{l}\text { Cobertura vegetal } \\
\text { y uso del suelo }\end{array}$ & Herbáceas & Pastos mejorados & Agricultura & Bosques \\
\hline Relieve & $3-5 \%$ & $>5 \%$ & & \\
\hline $\begin{array}{l}\text { Amenaza por } \\
\text { inundación }\end{array}$ & Sin amenaza & Sin amenaza & Sin amenaza & Amenaza alta \\
\hline Materiales del suelo & $\begin{array}{l}\text { Sedimentos mixtos, } \\
\text { areniscas y arcillas }\end{array}$ & $\begin{array}{l}\text { Areniscas o } \\
\text { arcillas }\end{array}$ & Arcillas o limo & $\begin{array}{l}\text { Suelos ácidos, } \\
\text { sulfáticos, presencia } \\
\text { de limo }\end{array}$ \\
\hline Textura del suelo & $\begin{array}{l}\text { Franco arcilloso arenoso, } \\
\text { textura mediana }\end{array}$ & $\begin{array}{l}\text { Franco arenoso o } \\
\text { franco arcilloso }\end{array}$ & Franco limoso & $\begin{array}{l}\text { Suelos arcillosos } \\
\text { limosos de textura fina }\end{array}$ \\
\hline $\begin{array}{l}\text { Fertilidad y acidez } \\
\text { del suelo }\end{array}$ & $\begin{array}{l}\text { Suelos fértiles, } \mathrm{pH} \\
\text { entre } 6 \text { y } 7\end{array}$ & Fertilidad media & & \\
\hline $\mathrm{pH}<6$ & $\begin{array}{l}\text { Alta acidez, } \mathrm{pH}<5 \text {, } \\
\text { fertilidad baja }\end{array}$ & $\begin{array}{l}\text { Suelos no } \\
\text { fértiles, } \mathrm{pH}<4\end{array}$ & & \\
\hline $\begin{array}{l}\text { Clasificación por } \\
\text { calidad del agua }\end{array}$ & $\begin{array}{l}\text { No contaminada } \\
\text { por hidrocarburos } \\
\text { ni agroquímicos }\end{array}$ & $\begin{array}{l}\text { Contaminada por } \\
\text { agroquímicos }\end{array}$ & $\begin{array}{l}\text { Dentro de } \\
\text { los rangos de } \\
\text { contaminación }\end{array}$ & $\begin{array}{l}\text { Contaminación } \\
\text { por hidrocarburos } \\
\text { y agroquímicos }\end{array}$ \\
\hline $\begin{array}{l}\text { Índice de } \\
\text { disponibilidad hídrica }\end{array}$ & 1 & $>0.5$ & $<0.5$ & 0 \\
\hline $\begin{array}{l}\text { Accesibilidad } \\
\text { a drenajes }\end{array}$ & $<1 \mathrm{~km}$ & & & $>50 \mathrm{~km}$ \\
\hline Accesibilidad a vías & $\begin{array}{l}\text { Acceso a } 1 \mathrm{~km} \text { con } \\
\text { pavimento }\end{array}$ & $\begin{array}{l}\text { Acceso a } 10 \mathrm{~km} \\
\text { con pavimento }\end{array}$ & Carreteable & Sin acceso \\
\hline $\begin{array}{l}\text { Densidad de } \\
\text { población }\end{array}$ & 0-10 hab/km2 & $>10 \mathrm{hab} / \mathrm{km} 2$ & & \\
\hline Distancia al mercado & $<100 \mathrm{~km}$ & Hasta 200 km & Hasta 1000 km & $>1000 \mathrm{~km}$ \\
\hline $\begin{array}{l}\text { Conflictos de } \\
\text { usos del suelo }\end{array}$ & Sin conflicto & Usos pecuarios & Usuarios agrícolas & $\begin{array}{l}\text { Con hidrocarburos, } \\
\text { asentamientos e } \\
\text { infraestructura }\end{array}$ \\
\hline
\end{tabular}


Finalmente se realizó la combinación de la calificación asignada a cada variable con los pesos de importancia mediante la ecuación matemática $(A P=C \times P)$ para dar un valor de aptitud; este procedimiento fue realizado utilizando el software ERDAS 8.4. Después de obtener los valores finales de la aptitud piscícola se generó el mapa final de aptitud en formato shp.
La estructura del modelo que se implementó para llegar a la aptitud piscícola de Castilla La Nueva lo muestra la figura 3, en este se plasma el proceso llevado a cabo con cada una de las variables para obtener como resultado final el mapa de aptitud de la zona.

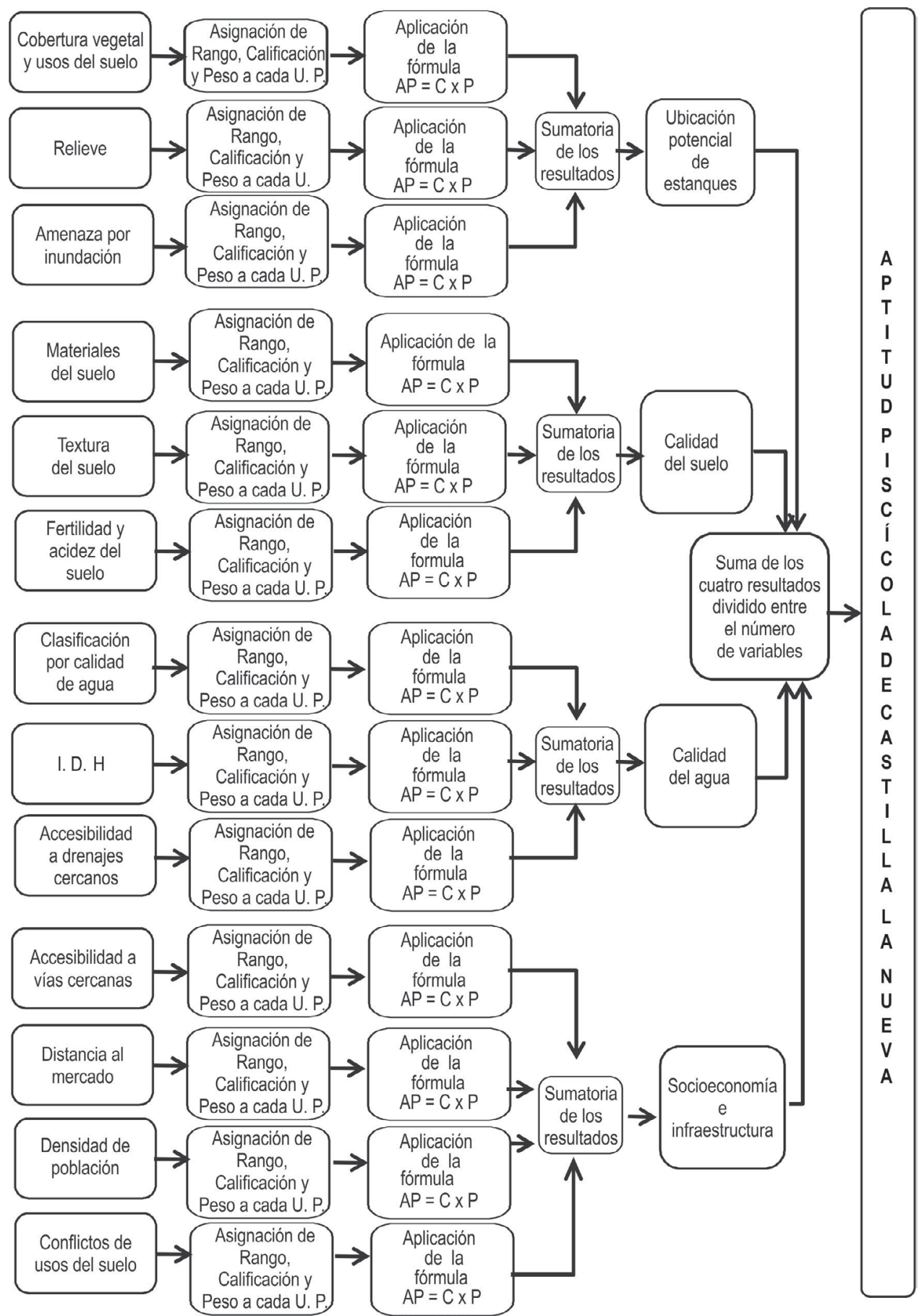

Figura 3. Modelo implementado en el estudio. 


\section{Resultados}

A través del uso de las herramientas SIG fue posible relacionar, modelar y representar los datos del municipio y la información sobre piscicultura de forma más precisa, obteniendo resultados más claros y resumidos para la toma de decisiones. En este caso las variables fueron relacionadas y modeladas bajo ciertos parámetros establecidos por los expertos para obtener la aptitud piscícola; de este modo los resultados obtenidos además de estar condicionados por las variables que se tuvieron en cuenta para el análisis espacial, también lo están por la interpretación de los datos realizada por los expertos en acuicultura.

El mapa de aptitud piscícola del municipio indica que para la época de estudio la mayor parte del municipio se encontraba dentro de un rango de aptitud alta (figura 4). Se puede observar que, para la época del estudio, el municipio presenta una aptitud que dentro del estudio está clasificada como apta; pero con algunas variaciones de los valores. Los valores de aptitud varían y difieren entre sí sólo entre 1 y 3\%. El área que tiene el valor más alto está favorecida por caracterís- ticas como un fácil acceso al mercado de comercialización, el no presentar conflictos de usos del suelo, accesibilidad a los drenajes, calidad del agua, entre otras. Las áreas No aptas para la piscicultura, estuvieron limitadas a zonas donde se presenta extracción de hidrocarburos y aquellas donde existe amenaza por inundación del río Guamal. Se puede decir que el resultado obtenido es producto de las propiedades con que cuenta el municipio, que además de ser bastante homogéneas, lo hacen apto para la actividad piscícola como son la gran riqueza hídrica, buen estado de las vías, relieve adecuado, entre otras.

\section{Discusión}

La primera consideración que debe hacerse para alcanzar las metas de sustentabilidad de una actividad, es conocer si el lugar en donde se piensa implantar la producción reúne las condiciones más apropiadas para el cultivo, para el caso de la piscicultura agua de buena calidad y cantidad, suelos aptos y que no impliquen grandes inversiones, apropiada infraestructura

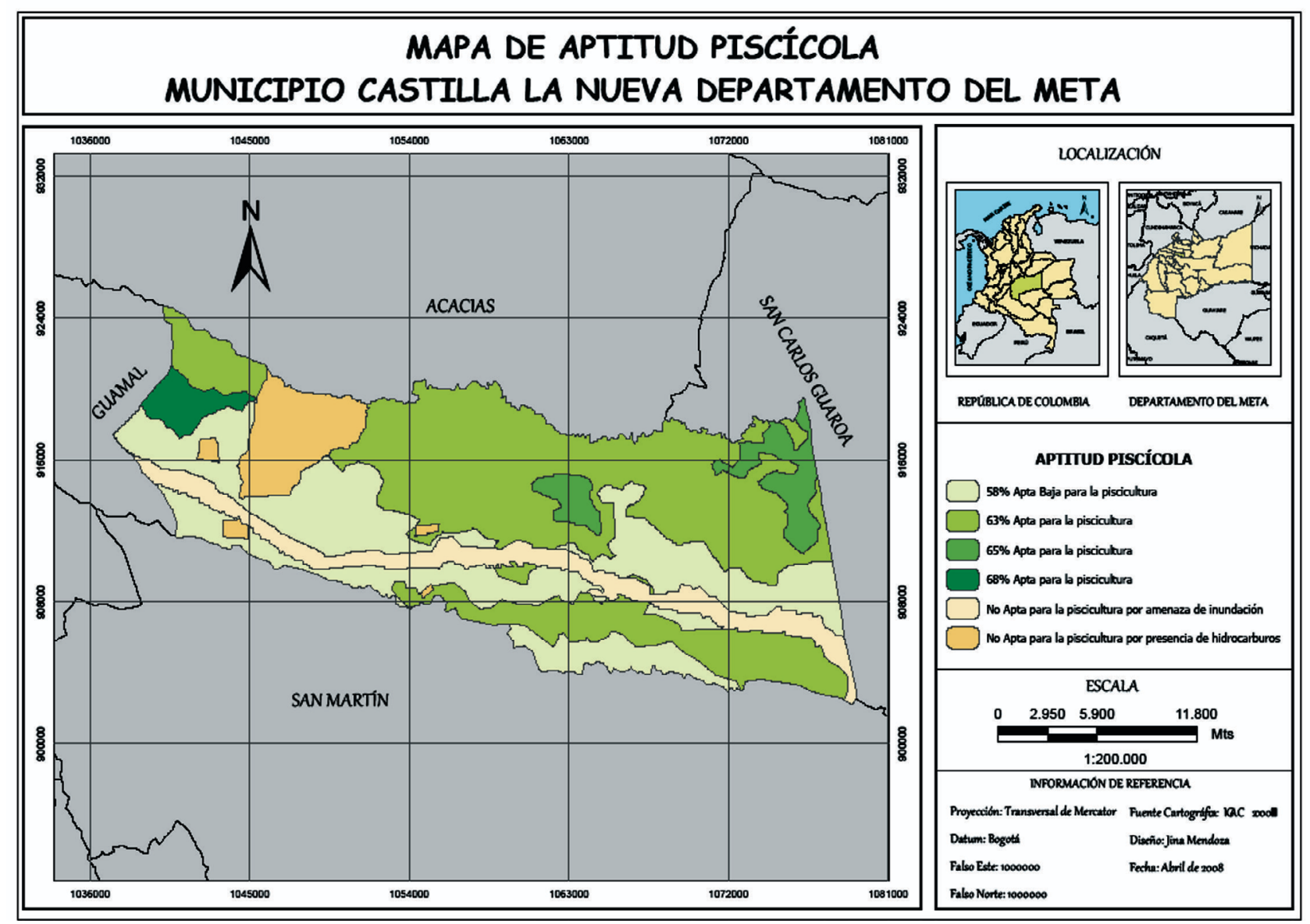

Figura 4. Aptitud Piscícola del Municipio Castilla La Nueva 
de apoyo a la actividad (vías, mercados, recursos humanos, entre otros).

Debido a lo anterior, este estudio identificó pocas áreas como No aptas para el desarrollo de la piscicultura. Castilla la Nueva cuenta con una extensión de 50.728 hectáreas de las cuales el 99\% está en el sector rural y el $1 \%$ en el sector urbano, con una población de 5.250 habitantes, distante de la capital del Meta 55 kilómetros, por carretera pavimentada y a 20 kilómetros de la troncal del Llano (IGAC, 2004), situación que confiere una alta aptitud para la variable de acceso. La cobertura hídrica en el municipio de Castilla la Nueva, está dada por tres grandes ríos: Guamal, Humadea y Orotoy, cada uno de los cuales forma su propia cuenca, característica que otorga gran favorabilidad a la actividad acuícola en el municipio. La única zona identificada con alto riesgo de inundación es la ribera del río Guamal.

Para fines administrativos el municipio de Castilla La Nueva se divide en cabecera municipal y 14 veredas, entre las cuales se destacan por su actividad piscícola las veredas Caño Grande, San Lorenzo, San Agustín, Betania, San Antonio y Cacayal, mediante estanques construidos específicamente para tal fin. Puesto que la piscicultura es una actividad que se encuentra establecida dentro del municipio, se espera que los resultados de este estudio sean la base para mejorar el manejo ambiental de la actividad piscícola en la zona y que se pueda integrar con otras actividades económicas de una forma ordenada y planificada. Tal como lo aseguraron Aguilar-Manjarrez et al., (2006), este mapa de aptitud, desarrollado con la ayuda de un SIGs, facilitará la correcta planificación y monitoreo de los futuros proyectos de inversión piscícola en el municipio de Castilla La Nueva. Uno de los problemas de la piscicultura en el país, es la falta de informaciones exactas sobre la disponibilidad de los recursos tierra, agua, humanos y económicos, lo que se traduce en equívocos en la selección de las áreas, surgimiento de problemas ambientales y conflictos con otros usuarios de los recursos (Kapetsky et al., 1998a, 1998b).

Este estudio es un ejemplo de los que se deben realizar para atender la debilidad reportada en el Diagnóstico del estado de la Acuicultura en Colombia (Merino et al., 2013), esta se refiere a la falta de información técnica y ambiental que sustente la vocación de cada región del país para la acuicultura. Este tipo de estudios deja claro que la planificación de una región requiere estar integrada con tecnologías que permitan tener una base de información espacial actualizada. Se debe destacar la importancia de los SIG con fines piscícolas, ya que permite involucrar aspectos que garantizan el óptimo desarrollo de la actividad a mediano y largo plazo.

\section{Agradecimientos}

Este estudio fue financiado por el proyecto código Código FMV-02-05 de la Universidad de Córdoba

\section{Referencias}

Aguilar-Manjarrez J, Bensch A, Carocci F, De Graaf G, Taconet M. Use of Geographic Information Systems (GIS) for Responsible Aquatic Resource Management. Fao Aquaculture Newsletter. 2006;35:13-19.

Aguilar-Manjarrez J, Nath SS. 1998. A strategic reassessment of fish farming potential in Africa. CIFA Technical paper. 32, FAO, Rome. http:www.fao.org:docrep:w8522e:w8522e00.htm.

Aguilar-Manjarrez J, Ross LG. Geographical information systems (GIS), environmental models for aquaculture development in Sinaloa state, Mexico. Aquaculture Int. 1995; 3:103-115.

Aguilar-Manjarrez J. 1992. Construction of a GIS for Tobasco State Mexico. Establishment of technical and social decision models for aquaculture development. MSc Thesis, University of Stirling, UK.

Chimowa M, Nugent C. 1993. A fisheries GIS for Zimbabwe: an initial analysis of the numbers, distribution and size of Zimbabwe's dams. AGRITEX Fisheries Unit-Technical Report.

Ecopetrol, 2004. Carta petrolera, noviembre 2003 - enero 2004. Revisada en Agosto de 2013 en http://www.ecopetrol.com.co/ especiales/carta_petrolera/empresa.htm

FAO, 2009. El Estado Mundial de la Pesca y la Acuicultura 2008. Departamento de Pesca y Acuicultura. Subdivisión de Políticas y apoyo en Materia de Publicación Electrónica. División de Comunicación. Roma, 2009. Sitio Web: http://www.fao.org

FAO. 1995. FAO Código de Conducta para la Pesca Responsable. Roma.

Giap DH, Yi Y, Yakupitiyage A. GIS for land evaluation for shrimp farming in Haiphong of Vietnam. Ocean Coast Manage. 2005;48(1):51-63.

IGAC - DPTO DEL META. 2004. El Meta: un territorio de oportunidades. Bogotá, D.C.

Kapetsky JM, Mcgregor L, Nanne EH. 1998a. A geographical information systems and satellite remote sensing to plan for aquaculture development: A FAO-UNEP/GRID cooperative study in Costa Rica. Fisheries Technical Paper (FAO), n 287 ISBN: 1988.92-5-102575, Roma.

Kapetsky JM, Hikk JM, Worthy LD. A geographical information system for catfish farming development. Aquaculture. 1998b;68:311-320.

Kapetsky JM, Hill JM, Worthy DL, Evans DL. Assessing potential for aquaculture development with a geographic information system. J World Aqua Soc. 1990;21:241-249. 
Kapetsky JM, Nath SS, 1997. A strategic assessment of the potential for freshwater fish farming in Latin America. COPESCAL Technical Paper N. 10. FAO, Rome.

Kapetsky JM, Travaglia C. 1995. Geographical information systems and remote sensing: an overview of their present and potential applications in aquaculture. In: Nambiar, K.P.P., Singh, T. (Eds.), AquaTech '94: Aquaculture Towards the 21st Century. INFOFISH, Kuala Lumpur, pp. 187-208.

Merino MC, Bonilla SP, Bages F. 2013. Diagnóstico del estado de la acuicultura en Colombia. Plan Nacional de Desarrollo de la Acuicultura Sostenible en Colombia AUNAP-FAO. Ministerio de Agricultura y Desarrollo Rural, Bogotá, 160p.

Nath SS, Bolte JP, Ross LG, Aguilar-Manjarrez J. Applications of geographical information systems (GIS) for spatial decision support in aquaculture. Aquacult. Eng. 2000;23:233-278.
Pardo-Carrasco S. 2006. Diagnóstico del estado ambiental y la elaboración de un modelo de gestión ambiental para la piscicultura del municipio de Castilla La Nueva (Meta, Colombia); Tesis de Doctorado (Ingeniería de Producción) Universidad Federal de Santa Catarina, Brasil.

Ross LG, Mendoza EA, Beveridge MCM. The application of geographical information systems to site selection for coastal aquaculture: an example based on salmonid cage culture. Aquaculture. 1993;112:165-178.

Scott PC, Cansado S, Ross LG. 1998. A GIS-assisted mollusc culture potential determination for Sepetiba Bay, Brazil. Paper presented at the GIS Planet '98 Conference, 7-11 September, 1998. Lisboa. Portugal.

Seiffert WQ. 2004. Cultivo de Camarões. In: Editora da UFSC. (Org.). Fundamentos de Aquicultura. Editora da UFSC, Florianópolis, SC, Brasil 